\title{
Symmetric Integration Rules for Hypercubes II. Rule Projection and Rule Extension
}

\author{
By J. N. Lyness
}

\begin{abstract}
A theory is described which facilitates the construction of highdimensional integration rules. It is found that, for large $n$, an $n$-dimensional integration rule of degree $2 t+1$ man be constructed requiring a number of function evaluations of order $2^{t} n^{t} / t$ !. In an example we construct a 15-dimensional rule of degree 9 which requires 52,701 function evaluations. The corresponding number for the product Gaussian is $3 \times 10^{10}$.
\end{abstract}

1. Introduction. The purpose of this paper is to introduce a theory of rule extension by which an $s$-dimensional rule of particular degree may be used to construct an $r$-dimensional rule of predetermined degree. This is a generalisation of the process which leads to a product rule. The particular feature of this process is that it is a linear process; once a set of nonlinear equations have been solved to obtain the $s$-dimensional rule, the higher-dimensional rule may be obtained by the application of linear algebraic formulae. In this paper we derive hitherto unpublished integration rules. The only nonlinear equations whose solution we assume are the Legendre equations, whose roots are the points used for function evaluation in the Gauss-Legendre quadrature formula (Gauss [1]).

Sections 2, 3, and 4 are devoted entirely to the theory of rule projection and extension and to consideration of the degree of the consequent rules. Sections 6 and 7 give specific types of rules derived by using this theory in a straightforward manner to extend the Gauss-Legendre quadrature formula. In Section 8 the rules obtained in Section 7 are shown to be of possible practical use and are used to establish a conjecture of Thacher [9].

In Part I (Lyness [5]) we introduced notation to describe integration rules. We assume without reference in this part the definitions of Part I and some of the results based on these definitions. In particular, the basic rule, expressed as a convolution product,

$$
\mathfrak{R}\left(\alpha_{1}, \alpha_{2}, \cdots, \alpha_{n}\right)=\mathfrak{R}\left(\alpha_{1}\right) * \mathfrak{R}\left(\alpha_{2}\right) \cdots * \mathfrak{R}\left(\alpha_{n}\right)=\prod_{i=1}^{n} \mathfrak{R}\left(\alpha_{i}\right),
$$

and the composite rule,

$$
R^{(n)}=\sum \xi_{i} \mathcal{R}^{(n)}
$$

are assumed together with the definition of degree and the result that the degree of a product rule $\left(R^{(1)}\right)^{n}$ is the same as that of the one-dimensional rule $R^{(1)}$.

In order to facilitate later generalisations, we consider a hypercube of edge 1 and so we set $a=\frac{1}{2}$ in the definitions of Part I. Also, for this reason we make no explicit use of the error coefficients $c_{2 s_{1} 2 s_{2}} \ldots(R)$. The use of $R_{i}{ }^{(n)}$ as in Section 4 of

Received May 8, 1964. This research was supported in part by U. S. Air Force Grant No. $62-400$ to the University of New South Wales. 
Part I in connection with cytolic integration is discontinued. Instead we use the superscript to indicate the dimension of a rule and a subscript to indicate its degree. $R_{t+1}^{(n)}$ is an $n$-dimensional integration rule of degree $2 t+1$.

2. Rule Projection. It is well known that an $n$-dimensional integration rule may be used to construct an $(n-1)$-dimensional integration rule by projection from the $n$-dimensional space to an $(n-1)$-dimensional space. For example, if we apply the basic three-dimensional rule $R(\alpha, \beta, \gamma)$ which uses 48 points to a function $f(x, y)$ independent of $z$, we find immediately that we obtain an identical result to that obtained if we were to apply the rule

$$
\frac{1}{3}(R(\beta, \gamma)+R(\gamma, \alpha)+R(\alpha, \beta))
$$

to the function $f(x, y)$. We describe this two-dimensional rule as the projection of $R(\alpha, \beta, \gamma)$ and write

$$
R(\alpha, \beta, \gamma) \Rightarrow P(R(\alpha, \beta, \gamma))=\frac{1}{3}(\Re(\beta, \gamma)+R(\gamma, \alpha)+R(\alpha, \beta)),
$$

the symbol $\Rightarrow$ being read as 'projects onto' and the operator $\odot$ as 'the projection of'.

We define the projection of an $r$-dimensional basic rule onto an $(r-1)$-dimensional rule as follows:

$$
\mathcal{R}\left(\alpha_{1}, \alpha_{2}, \cdots, \alpha_{r}\right) \Rightarrow \mathcal{P}\left(\Re\left(\alpha_{1}, \alpha_{2}, \cdots, \alpha_{r}\right)\right)=\frac{1}{r} \sum_{j=1}^{r} \prod_{i=1 ; i \neq j}^{r} R\left(\alpha_{i}\right) .
$$

It follows by inspection that if $f$ is a function of $r-1$ variables, then

$$
\mathfrak{R}^{(r)} f\left(x_{1}, x_{2}, \cdots, x_{r-1}\right)=\left(\mathcal{P}\left(\mathbb{R}^{(r)}\right)\right) f\left(x_{1}, x_{2}, \cdots, x_{r-1}\right) .
$$

We define the projection of a composite rule $R^{(r)}$ in terms of the projection of basic rules $\mathcal{Q}^{(r)}$ by

$$
R^{(r)}=\sum \xi_{i} \mathcal{R}_{i}{ }^{(r)} \Rightarrow \mathcal{P}\left(R^{(r)}\right)=\sum \xi_{i} \mathcal{P}\left(\mathcal{R}_{i}{ }^{(r)}\right)
$$

and we find, as for the basic rules, that if $f$ is a function of $r-1$ variables, then

$$
R^{(r)} f\left(x_{1}, x_{2}, \cdots, x_{r-1}\right)=\left(\rho\left(R^{(r)}\right)\right) f\left(x_{1}, x_{2}, \cdots, x_{r-1}\right) .
$$

For convenience, we introduce a zero-dimensional rule $\mathscr{g}$ with the property

$$
R(\alpha) \Rightarrow \mathcal{P}(R(\alpha))=g
$$

and

$$
\mathcal{g} * \mathbb{R}^{(r)}=\mathbb{R}^{(r)} .
$$

Making use of this symbol $g$ where necessary, we may establish, using straightforward algebra, that

$$
\odot\left(\eta_{1} R_{1}{ }^{(r)}+\eta_{2} R_{2}{ }^{(r)}\right)=\eta_{1} \odot\left(R_{1}{ }^{(r)}\right)+\eta_{2} \odot\left(R_{2}{ }^{(r)}\right)
$$

and

$$
\mathcal{P}\left({R_{1}}^{(s)} *{R_{2}}^{(t)}\right)=\frac{1}{s+t}\left\{s\left(\mathcal{P}\left({R_{1}}^{(s)}\right)\right) *{R_{2}}^{(t)}+t R_{1}{ }^{(s)} * \mathcal{P}\left(R_{2}{ }^{(t)}\right)\right\}
$$


Applying this to a product rule, we find

$$
\mathcal{P}\left(\left(R^{(1)}\right)^{n}\right)=\left(R^{(1)}\right)^{n-1} \text {. }
$$

We now extend slightly the definition of projection. We define the $s$-dimensional projection $R^{(s)}$ of an $r$-dimensional rule $R^{(r)}(0 \leqq s<r)$ as the result of $r-s$ successive projections of $R^{(r)}$. For example, the one-dimensional projection of the three-dimensional rule $R(\alpha, \beta, \gamma)$ is $\rho(\rho(R(\alpha, \beta, \gamma)))$ and, using (2.2) twice, we see that

$$
\mathbb{R}(\alpha, \beta, \gamma) \Rightarrow P(P(\mathcal{R}(\alpha, \beta, \gamma)))=\frac{1}{3}(\mathbb{R}(\alpha)+\mathbb{R}(\beta)+\mathbb{R}(\gamma)) .
$$

(The symbol $\Rightarrow$ is used to describe any number of projections but the operator $\beta$ has been defined to project onto a dimension lower by one than the dimension of the rule on which it operates.) It follows that all rules $R^{(r)}$ project onto $g$,

$$
\begin{aligned}
& R^{(r)} \Rightarrow g, \\
& \left(R^{(1)}\right)^{n} \Rightarrow R^{(1)},
\end{aligned}
$$

and that if $R^{(r)} \Rightarrow R^{(s)}$, then

$$
R^{(r)} f\left(x_{1}, x_{2}, \cdots, x_{8}\right)=R^{(s)} f\left(x_{1}, x_{2}, \cdots, x_{s}\right) .
$$

3. Rule Extension. The technique of rule projection described in the previous section is not by itself a powerful technique for deriving integration rules. It provides a method of deriving an $(r-1)$-dimensional rule if an $r$-dimensional rule is known. In general, the opposite situation prevails, i.e., we may know many one- or two-dimensional rules and be interested in determining rules in higher dimensions. We consider in this section an inverse process to that of rule projection. We term this process 'rule extension'.

A particular case of rule extension is the $n$-dimensional product rule, which is well known. A method for obtaining an integration formula for an $(n+1)$-dimensional cone if a formula is known for its $n$-dimensional base is given by Hammer, Marlowe and Stroud [2]. Also Stroud [8] shows how to construct a formula of degree 3 for the cartesian product $R_{r} \times R_{s}$ of an $r$-dimensional region $R_{r}$ and an $s$-dimensional region $R_{s}$, provided that formulas of degree 3 are known for $R_{r}$ and $R_{s}$. In both of these cases, the formulas are of different types than those discussed here.

To illustrate the method we suppose that we wish to find a three-dimensional extension of the two-dimensional basic rule $R(\alpha, \beta)$. It is convenient to introduce an arbitrary coordinate $\gamma$ which we term the extension coordinate. We then look for a three-dimensional rule $R^{(3)}$ in the form

$$
R^{(3)}=\lambda_{1} R(\alpha, \beta, \gamma)+\lambda_{2} R(\beta, \gamma, \gamma)+\lambda_{3} R(\alpha, \gamma, \gamma)+\lambda_{4} R(\gamma, \gamma, \gamma) .
$$

We make use of the projections

$$
\begin{aligned}
& P(R(\alpha, \beta, \gamma))=\frac{1}{3} R(\beta, \gamma)+\frac{1}{3} R(\gamma, \alpha)+\frac{1}{3} R(\alpha, \beta) . \\
& P(R(\beta, \gamma, \gamma))=\frac{1}{3} R(\gamma, \gamma)+\frac{2}{3} R(\beta, \gamma), \\
& P(R(\alpha, \gamma, \gamma))=\frac{1}{3} R(\gamma, \gamma)+\frac{2}{3} \mathbb{R}(\gamma, \alpha), \\
& P(R(\gamma, \gamma, \gamma))=R(\gamma, \gamma) .
\end{aligned}
$$


The condition that $R^{(3)}$ projects onto $R(\alpha, \beta)$ then leads to four equations in the four unknowns $\lambda_{1}, \lambda_{2}, \lambda_{3}$, and $\lambda_{4}$. The solution is $\lambda_{1}=3, \lambda_{2}=\lambda_{3}=-\frac{3}{2}, \lambda_{4}=1$, so the rule $R^{(3)}$ is

$$
R^{(3)}=3 \mathfrak{R}(\alpha, \beta, \gamma)-\frac{3}{2} \mathfrak{R}(\beta, \gamma, \gamma)-\frac{3}{2} \mathfrak{R}(\alpha, \gamma, \gamma)+\mathfrak{R}(\gamma, \gamma, \gamma)
$$

We term $R^{(3)}$ the three-dimensional extension of $R(\alpha, \beta)$ using extension coordinate $\gamma$ and write it in terms of an extension operator $E_{2}{ }^{3}(\gamma)$

$$
R^{(3)}=E_{2}{ }^{3}(\gamma) \mathcal{R}(\alpha, \beta) .
$$

It should be noted that the extension coordinate $\gamma$ may be any positive number or zero. Moreover, $R^{(3)}$ is not defined as the only three-dimensional rule which projects onto $R(\alpha, \beta)$, but merely one of the form (2.1). The choice of $\gamma$ sometimes is based on the number of points $\nu\left(R^{(3)}\right)$ which the rule $R^{(3)}$ requires (see Section 5 of Part I). In this case, if $\alpha \neq \beta$ and neither is zero, we find $\nu\left(R^{(3)}\right)$ is 25,24 or 88 according as $\gamma$ is zero, one of $\alpha$ or $\beta$, or some other value. Thus, in the practice of rule extension, it is usual to choose as $\gamma$ either a coordinate which is already present, in this case $\alpha$ or $\beta$, or else the value zero. lows:

We now define the $(s+1)$-dimensional rule $E_{s}{ }^{s+1}(\gamma) \mathcal{R}\left(\alpha_{1}, \alpha_{2}, \cdots, \alpha_{s}\right)$ as fol-

$$
E_{s}{ }^{s+1}(\gamma) \mathcal{R}\left(\alpha_{1}, \alpha_{2}, \cdots, \alpha_{s}\right)
$$

$$
\begin{aligned}
& =B_{0}\{R(\gamma)\}^{s+1}+B_{1}\{R(\gamma)\}^{s} *\left\{R\left(\alpha_{1}\right)+\mathscr{R}\left(\alpha_{2}\right)+\cdots+R\left(\alpha_{s}\right)\right\} \\
& +B_{2}\{\mathcal{R}(\gamma)\}^{s-1} * \sum_{i=1}^{s} \sum_{j=i+1}^{s} R\left(\alpha_{i}\right) * \mathcal{R}\left(\alpha_{j}\right)+\cdots+B_{s} R(\gamma) * \prod_{j=1}^{s} R\left(\alpha_{j}\right),
\end{aligned}
$$

where

$$
B_{t}=\frac{(-1)^{s-t}(s+1)}{s-t+1}
$$

It may be verified, by direct application of the rules for the projection of composite rules given in the previous section, that

$$
\begin{aligned}
E_{s}^{s+1}(\gamma) \mathcal{R}\left(\alpha_{1}, \alpha_{2}, \cdots, \alpha_{s}\right) \Rightarrow \mathcal{P}\left(E_{s}^{s+1}(\gamma) \mathcal{R}\left(\alpha_{1}, \alpha_{2}, \cdots, \alpha_{s}\right)\right) \\
=\mathbb{R}\left(\alpha_{1}, \alpha_{2}, \cdots, \alpha_{s}\right) .
\end{aligned}
$$

The $(s+1)$-dimensional extension of an $s$-dimensional composite rule $R^{(s)}$ is defined in terms of the extension of basic rules as follows. If

$$
R^{(s)}=\sum \xi_{i} \mathcal{R}_{i}{ }^{(s)} \text {. }
$$

then

$$
E_{s}{ }^{s+1}(\gamma) R^{(s)}=\sum \xi_{i} E_{s}{ }^{s+1}(\gamma) R_{i}{ }^{(s)} .
$$

It is trivial to show that

$$
E_{s}{ }^{s+1}(\gamma) R^{(s)} \Rightarrow \odot\left(E_{s}{ }^{s+1}(\gamma) R^{(s)}\right)=R^{(s)} .
$$

Using equations (3.5) and (3.8) $r-s$ times, and using the same value of $\gamma$ 
each time, we may construct an $r$-dimensional rule $R^{(r)}$ which we term the $r$-dimensional extension of $R^{(s)}$ using extension coordinate $\gamma$. This is defined as

$$
R^{(r)}=E_{r-1}^{r}(\gamma) E_{r-2}^{r-1}(\gamma) \cdots E_{s}{ }^{s+1}(\gamma) R^{(s)} \equiv E_{s}{ }^{r}(\gamma) R^{(s)} .
$$

It follows from equations (3.8) and (3.10) that

$$
E_{s}{ }^{r}(\gamma)\left(\sum \xi_{i}{R_{i}}^{(s)}\right)=\sum \xi_{i} E_{s}{ }^{r}(\gamma) \mathbb{R}_{i}{ }^{(s)} .
$$

Iteration of equation (3.5) leads to the formula,

$$
\begin{aligned}
& E_{s}{ }^{r}(\gamma) \mathcal{R}\left(\alpha_{1}, \alpha_{2}, \cdots, \alpha_{s}\right) \\
& =A_{0}\{\mathbb{R}(\gamma)\}^{r}+A_{1}\{\mathbb{R}(\gamma)\}^{r-1} *\left\{\mathbb{R}\left(\alpha_{1}\right)+\mathscr{R}\left(\alpha_{2}\right)+\cdots+\mathbb{R}\left(\alpha_{s}\right)\right\} \\
& +A_{2}\{R(\gamma)\}^{r-2} * \sum_{i=1}^{s} \sum_{j=i+1}^{s} R\left(\alpha_{i}\right) * R\left(\alpha_{j}\right)+\cdots+A_{s}\{R(\gamma)\}^{r-s} * \prod_{j=1}^{s} R\left(\alpha_{j}\right) \text {, }
\end{aligned}
$$

where

$$
A_{t}=\frac{(-1)^{s-t} r !(r-s)}{(r-s) ! s !(r-t)}
$$

It is sometimes convenient to extend product rules. Letting $R^{(1)}$ be a one-dimensional rule and using the techniques of this section, we find without difficulty that

$$
E_{s}^{r}(\gamma)\left(R^{(1)}\right)^{s}=\frac{r !}{s !(r-s-1) !} \sum_{t=0}^{s} \frac{(-1)^{t-s}}{r-t}\left(\begin{array}{l}
s \\
t
\end{array}\right)\{\Re(\gamma)\}^{r-t} *\left(R^{(1)}\right)^{t}
$$

If $s=1$, this becomes

$$
E_{1}{ }^{r}(\gamma) R^{(1)}=-(n-1)\{R(\gamma)\}^{r}+n\{R(\gamma)\}^{r-1} * R^{(1)} .
$$

Any rule may be expressed as a linear combination of product rules. Thus, in certain cases, (3.13) may be used as an alternative to (3.12) to reduce the amount of computation in extending a rule.

Simple examples of rule extension include the following result:

$$
E_{s}{ }^{r}(\gamma)\{R(\gamma)\}^{s}=\left\{(R(\gamma)\}^{r} .\right.
$$

However, this is the only case in which the extension operator $E_{r}^{8}(\gamma)$ operating on a product rule results in another product rule. The situation is illustrated by considering two-dimensional extensions of Simpson's rule:

$$
R_{s}=\frac{2}{3} \Re(0)+\frac{1}{3} R(1) .
$$

We find that

$$
\left(R_{s}\right)^{2}=\frac{4}{3} E_{1}^{2}(0) R_{s}-\frac{1}{3} E_{1}^{2}(1) R_{s},
$$

and the corner and vertex rule in two dimensions,

$$
R_{\mathrm{cv}}^{(2)}=\frac{2}{3}\left\{(R(0)\}^{2}+\frac{1}{3}\{\Re(1)\}^{2}=2 E_{1}^{2}(0) R_{s}-E_{1}{ }^{2}(1) R_{s} .\right.
$$

Each of the different rules $\left(R_{s}\right)^{2}, R_{\mathrm{cv}}^{(2)}, E_{1}{ }^{2}(\gamma) R_{s}$ project onto $R_{s}$.

4. The Degree of an Extended Rule. In Part I we defined the operator $I$ to be the integration operator. It is convenient to re-define the integration operator in $r$ 
dimensions as follows

$$
I^{(r)} f=\int_{-\frac{1}{2}}^{\frac{1}{2}} \int_{-\frac{1}{2}}^{\frac{1}{2}} \cdots \int_{-\frac{1}{2}}^{\frac{1}{2}} f d x_{1} d x_{2} \cdots d x_{r},
$$

where $f$ is a function of at most the $r$ variables $x_{1}, x_{2}, \cdots, x_{r}$. This definition corresponds to the definition in Part I if we set $a=\frac{1}{2}$ in that definition. If $f$ is a function of only $s$ of the $r$ variables, we see that

$$
I^{(r)} f\left(x_{1}, x_{2}, \cdots, x_{s}\right)=I^{(s)} f\left(x_{1}, x_{2}, \cdots, x_{s}\right),
$$$$
r>s \text {. }
$$

An $n$-dimensional integration rule $R_{t+1}^{(n)}$ of degree $2 t+1$ is one which integrates correctly all polynomials of degree $2 t+1$ or less in the $n$ variables. We restrict ourselves to symmetric integration rules only. One consequence is that any polynomial which is odd in any variable is integrated to its correct value zero. Moreover, if the rule is constructed so that it integrates correctly a polynomial $x_{1}{ }^{n_{1}} x_{2}{ }^{n_{2}} \ldots$ $x_{n}{ }^{n_{n}}$, then it automatically integrates correctly the polynomial $x_{1}{ }^{m_{1}} x_{2}{ }^{m_{2}} \cdots x_{n}{ }^{m_{n}}$, where $m_{1} m_{2} \cdots m_{n}$ are a permutation of the integers $n_{1} n_{2} \cdots n_{n}$. Thus, a necessary and sufficient condition that $R_{t+1}^{(n)}$ is of degree $2 t+1$ is

$$
R_{t+1}^{(n)} f=I^{(n)} f \text { when } f \in \Phi_{t+1}^{(n)},
$$

where $\Phi_{t+1}^{(n)}$ is a finite set of functions defined by

$$
\begin{aligned}
& \Phi_{t+1}^{(n)}=\left\{x_{1}^{{ }^{n_{1}}} x_{2}{ }^{n_{2}} \cdots x_{n}{ }^{n_{n}} \text { such that the } n_{i}\right. \text { are non-negative even } \\
& \left.\qquad \text { integers, } n_{1} \geqq n_{2} \geqq \cdots \geqq n_{n}, \text { and } \sum_{i=1}^{n} n_{i}<2 t+1\right\} .
\end{aligned}
$$

It is convenient to establish subsets of this set $\Phi_{t+1}^{(n)}$; we construct separate subsets $\phi_{t+1}^{m}$ which contain only polynomials in exactly $m$ variables. Thus

$$
\begin{aligned}
\phi_{t+1}^{m}=\left\{x_{1}{ }^{n_{1}} x_{2}{ }^{n_{2}} \cdots x_{m}{ }^{n_{m}} \text { such that the } n_{i}\right. \text { are non-negative even } \\
\left.\quad \text { integers, } n_{1} \geqq n_{2} \geqq \cdots \geqq n_{m}>0 \text {, and } \sum_{i=1}^{n} n_{i}<2 t+1\right\} .
\end{aligned}
$$

An example of these sets $(t=4)$ is

$$
\begin{aligned}
\phi_{5}{ }^{0} & =\{1\}, \\
\phi_{5}{ }^{1} & =\left\{x_{1}{ }^{2}, x_{1}{ }^{4}, x_{1}{ }^{6}, x_{1}{ }^{8}\right\}, \\
\phi_{5}{ }^{2} & =\left\{x_{1}{ }^{2} x_{2}{ }^{2}, x_{1}{ }^{4} x_{2}{ }^{2}, x_{1}{ }^{6} x_{2}{ }^{2}, x_{1}{ }^{4} x_{2}{ }^{4}\right\}, \\
\phi_{5}{ }^{3} & =\left\{x_{1}{ }^{2} x_{2}{ }^{2} x_{3}{ }^{2}, x_{1}{ }^{4} x_{2}{ }^{2} x_{3}{ }^{2}\right\}, \\
\phi_{5}{ }^{4} & =\left\{x_{1}{ }^{2} x_{2}{ }^{2} x_{3}{ }^{2} x_{4}{ }^{2}\right\}, \\
\phi_{5}{ }^{m} & =\{\},
\end{aligned}
$$$$
m \geqq 5 \text {. }
$$

These twelve polynomials have to be integrated exactly by any $n>4$ dimensional rule $R_{5}{ }^{(n)}$ of degree 9. Moreover, if $R^{(n)}$ satisfies the twelve equations $R^{(n)} f=I^{(n)} f$ when $f$ is one of these twelve functions, then $R^{(n)}$ is of degree 9 . 
Trivial consequences of these definitions are

$$
\begin{array}{lr}
\Phi_{t+1}^{(m)}=\Phi_{t+1}^{(m-1)} \cup \phi_{t+1}^{m}, & \\
\Phi_{t+1}^{(n)}=\phi_{t+1}^{0} \cup \phi_{t+1}^{1} \cup \ldots \cup \phi_{t+1}^{n}, & \\
\phi_{t+1}^{m}=\{\}, & m \geqq t+1, \\
\Phi_{t+1}^{(t)}=\Phi_{t+1}^{(t+p)} & p=0,1,2, \cdots, \\
\phi_{t+p}^{r} \supseteq \phi_{t}^{r} & p=0,1,2, \cdots .
\end{array}
$$

Using (4.7) we see that

$$
\Phi_{t+1}^{(m+p)} \supseteq \Phi_{t+1}^{(m)}
$$$$
p=0,1,2, \cdots
$$

Moreover, if $n<t$, we may use (4.11) in the form

$$
\phi_{t+1}^{r} \supseteq \phi_{n+1}^{r},
$$

$$
n<t
$$

together with (4.8) to establish

$$
\Phi_{t+1}^{(n)} \supseteq \phi_{n+1}^{0} \cup_{\phi_{n+1}^{1}}^{1} \cup \ldots \cup_{\phi_{n+1}^{n},} \quad n<t .
$$

The right-hand side of (4.14) is $\Phi_{n+1}^{(n)}$ and, using this together with (4.10), we find

$$
\Phi_{t+1}^{(n)} \supseteq \Phi_{n+1}^{(n)}=\Phi_{n+1}^{(n+p)} \quad n<t, p=0,1,2, \cdots
$$

We make explicit use of (4.7), (4.10), (4.12), and (4.15) in this section and in Section 7 .

We now establish the following Theorems: $R^{(r)}$.

TheOREM 4.1. If $R^{(r)} \Rightarrow R^{(s)}$, the degree of $R^{(s)}$ is at least as great as the degree of

Proof. If $R^{(r)}$ is of degree $2 t+1$, then

$$
R^{(r)} f=I^{(r)} f, \text { when } f \in \Phi_{\imath+1}^{(r)}
$$

but if $s<r$, equation (4.12) states that

$$
\Phi_{t+1}^{(s)} \subseteq \Phi_{t+1}^{(r)}
$$

Thus

$$
R^{(r)} f=I^{(r)} f, \text { when } f \in \Phi_{t+1}^{(s)} .
$$

All elements of $\Phi_{t+1}^{(s)}$ are functions of $s$ or fewer variables. Thus, using (2.14) and (4.2),

$$
\begin{aligned}
& R^{(r)} f=R^{(s)} f, \text { when } f \in \Phi_{i+1}^{(s)}, \\
& I^{(r)} f=I^{(s)} f, \text { when } f \in \Phi_{t+1}^{(s)} .
\end{aligned}
$$

Hence,

$$
R^{(s)} f=I^{(s)} f, \text { when } f \in \Phi_{t+1}^{(s)} .
$$

This is the condition that $R^{(s)}$ is of degree $2 t+1$ and so establishes the Theorem 4.1 .

THEOREM 4.2. If $R^{(r)} \Rightarrow R^{(s)}$ and $R^{(s)}$ is of degree $2 t+1$, the degree of $R^{(r)}$ is at least $2 t^{\prime}+1$, where $t^{\prime}=\min (s, t)$. 
Proof.

$$
R^{(s)} f=I^{(s)} f \text { when } f \in \Phi_{l+1}^{(s)} .
$$

Using (2.14) and (4.2),

$$
\begin{aligned}
& R^{(s)} f=R^{(r)} f, \text { when } f \in \Phi_{i+1}^{(s)}, \\
& I^{(s)} f=I^{(r)} f, \text { when } f \in \Phi_{i+1}^{(s)} .
\end{aligned}
$$

Hence,

$$
R^{(r)} f=I^{(r)} f, \text { when } f \in \Phi_{t+1}^{(s)} .
$$

If $s \geqq t,(4.10)$ states

$$
\Phi_{t+1}^{(r)}=\Phi_{l+1}^{(s)}
$$

for $s \geqq t$

Thus

$$
R^{(r)} f=I^{(r)} f, \text { when } f \in \Phi_{t+1}^{(r)}, \quad s \geqq t .
$$

Hence, the degree of $R^{(r)}$ is $2 t+1$. If $s<t$, (4.15) states

$$
\Phi_{t+1}^{(s)} \supseteq \Phi_{s+1}^{(s)}=\phi_{s+1}^{(r)}, \quad s<t .
$$

Thus,

$$
R^{(r)} f=I^{(r)} f, \text { when } f \in \Phi_{s+1}^{(r)}, \quad s<t .
$$

Hence, the degree of $R^{(r)}$ is $2 s+1$.

In both cases the degree of $R^{(r)}$ is $2[\min (s, t)]+1$. This establishes Theorem 4.2 .

5. Construction of High-Dimensional Low-Degree Rules. Theorems 4.1 and 4.2 provide directly a method for generating integration rules. Once the tedious task of calculating an $s$-dimensional integration rule $R_{t+1}^{(s)}$ of degree $2 t+1$ has been completed, we may construct $n>s$ dimensional rules of degree $2 t^{\prime}+1$, where $t^{\prime}=\min (s, t)$, merely by applying the formulae of Section 3 . This may be done in a wide variety of ways. We may simply calculate the rule $E_{s}{ }^{n}(\gamma) R_{t+1}^{(s)}$, which gives us a one-parameter system of such rules. Instead, we may proceed as follows. We may express $R_{t+1}^{(s)}$ in terms of its constituent basic rules and extend each basic rule using, possibly, a different extension parameter for each extension. This may be expressed as follows:

$$
\begin{aligned}
& R_{i+1}^{(s)}=\sum_{i=1}^{p} \xi_{i} \mathbb{R}_{i}^{\left({ }^{(s)}\right.}, \\
& R_{t^{\prime}+1}^{(n)}=\sum_{i=1}^{p} \xi_{i} E_{n-1}^{n}\left(\gamma_{i, n}\right) E_{n-2}^{n-1}\left(\gamma_{i, n-1}\right) \cdots E_{s}{ }^{s+1}\left(\gamma_{i, s+1}\right) \mathbb{R}_{i}^{(s)} .
\end{aligned}
$$

It is easy to verify that

$$
R_{t^{\prime}+1}^{(n)} \Rightarrow R_{t+1}^{(s)} .
$$

Thus $R_{t^{\prime}+1}^{(n)}$ is an $n$-dimensional rule of degree $2 t^{\prime}+1$, where $t^{\prime}=\min (s, t)$, which has $(n-s) p$ arbitrary parameters $\gamma_{i j}$. These parameters may be chosen with any 
end in view, but they are normally chosen to minimise some component of the error. In the examples in Sections 6 and 7 we choose $\gamma=0$, as this has the effect of keeping the number of function evaluations $\nu\left(R_{t^{\prime}+1}^{(n)}\right)$ reasonably small.

The systematic construction of $n$-dimensional integration rules using this technique and powerful generalisations is being carried out. In this paper we limit ourselves to two simple and practical illustrations.

6. The Rules $E_{t}{ }^{n}(0)\left(\left(G_{t+1}\right)^{t}\right)$. The one-dimensional $(t+1)$-point Gauss-Legendre quadrature formula of degree $2 t+1$ is widely discussed in many books on numerical integration (Gauss [1]). We write it as follows:

$$
G_{t+1}=\sum_{i=1}^{\lfloor(t+1) / 2\}} \zeta_{i} R\left(\beta_{i}\right) .
$$

The values of $\zeta_{i}$ and $\beta_{i}$ are readily available in the literature (see for example: Kopal [4]).

The $n$-dimensional product Gaussian $\left(G_{t+1}\right)^{n}$ is, for all values of $t$ and $n$ both greater than 3 , the most efficient in terms of function evaluations of the hitherto published integration rules. The number of points required by this rule is

$$
\nu\left(\left(G_{t+1}\right)^{n}\right)=(t+1)^{n} .
$$

However, we may construct the rule

$$
E_{t}{ }^{n}(0)\left(G_{t+1}\right)^{t} \Rightarrow\left(G_{t+1}\right)^{t} .
$$

Since $\left(G_{t+1}\right)^{t}$ is a product rule, it has the same degree as $G_{t+1}$, namely, $2 t+1$. Using Theorem 4.2, the degree of

$$
E_{t}^{n}(0)\left(\left(G_{t+1}\right)^{t}\right)
$$

is seen to be $2 t+1$. The number of function evaluations required by this rule is easily calculated using the formulae of Section 5 of Part I. It appears that

$$
\begin{aligned}
\nu\left(E_{t}{ }^{n}(0)\left(G_{t+1}\right)^{t}\right)= & 1+t\left(\begin{array}{l}
n \\
1
\end{array}\right)+t^{2}\left(\begin{array}{l}
n \\
2
\end{array}\right)+\cdots+t^{t}\left(\begin{array}{l}
n \\
t
\end{array}\right) \quad(t \text { even }) \\
= & 1+(t+1)\left(\begin{array}{l}
n \\
1
\end{array}\right)+(t+1)^{2}\left(\begin{array}{l}
n \\
2
\end{array}\right) \\
& +\cdots+(t+1)^{t}\left(\begin{array}{l}
n \\
t
\end{array}\right) \quad(t \text { odd }) .
\end{aligned}
$$

In both cases this is a polynomial of degree $t$ in $n$; the leading term is either $\left(t^{t} / t !\right) n^{t}$ or $\left((t+1)^{t} / t !\right) n^{t}$.

If $n>t$, this rule is usually a considerable improvement (from the point of view of the required number of points) on the presently available product Gaussian $\left(G_{t+1}\right)^{n}$ of the same degree. In fact, if $t$ is even, the points used by this rule, $E_{t}{ }^{n}(0)\left(G_{t+1}\right)^{t}$, constitute a subset of the points used by $\left(G_{t+1}\right)^{n}$, so

$$
\nu\left(E_{t}{ }^{n}(0)\left(G_{t+1}\right)^{t}\right)<\nu\left(\left(G_{t+1}\right)^{n}\right) \quad(t \text { even }) .
$$

For odd $t$ inequality (6.5) is not necessarily true, though it is certainly true if $t$ is 
TABLE 1

Number of Points Required by Certain 15-Dimensional Integration Rules

\begin{tabular}{c|c|c|c|r|r}
\hline Degree & $t$ & $\nu\left(G_{t+1}^{15}\right)$ & $\nu\left(E_{t}^{15}(0) G_{t+1}^{t}\right)$ & $\nu\left(\bar{G}_{t+1}^{(15)}\right)$ & Lower bound \\
\cline { 2 - 4 } & 1 & 32,768 & 31 & & 30 \\
5 & 2 & $1.43 \times 10^{7}$ & 451 & & 420 \\
7 & 3 & $1.07 \times 10^{9}$ & 30,861 & 5,381 & 3,640 \\
9 & 4 & $3.05 \times 10^{10}$ & 380,301 & 52,701 & 21,840 \\
\hline
\end{tabular}

The rule $\bar{G}_{u+1}^{(15)}$ is defined in equation (7.16). The lower bound which applies to a symmetric rule is derived in a later publication.

much smaller than $n$. The situation is illustrated in Table 1 in which the number of points required by certain fifteen-dimensional rules are listed.

The only published rule of the form $E_{t}{ }^{n}(0)\left(\left(G_{t+1}\right)^{t}\right)$ is the first nontrivial case $t=2$. In this case, with $\beta=\sqrt{ } \frac{3}{5}$ we have

$$
\begin{aligned}
G_{3} & =\frac{4}{9} R(0)+\frac{5}{9} R(\beta), \\
\left(G_{3}\right)^{2} & =\frac{16}{81} R(0,0)+\frac{40}{81} \mathfrak{R}(0, \beta)+\frac{25}{81} R(\beta, \beta) .
\end{aligned}
$$

Substituting $G_{3}$ for $R^{(1)}$ into expression (3.13), we find

$$
\begin{aligned}
E_{2}{ }^{n}(0)\left(G_{3}\right)^{2}=\frac{n(n-1)(n-2)}{2} & \left\{\frac{1}{n-2}\{\Re(0)\}^{n-2} *\left\{G_{3}\right\}^{2}\right. \\
& \left.-\frac{2}{n-1}\{\Re(0)\}^{n-1} * G_{3}+\frac{1}{n}\{R(0)\}^{n}\right\} .
\end{aligned}
$$

Finally, substituting for $G_{3}$ and $G_{3}{ }^{2}$ and collecting together terms, we find

$$
\begin{aligned}
& E_{2}{ }^{n}(0)\left(G_{3}\right)^{2}=\frac{1}{162}\left\{\left(25 n^{2}-115 n+162\right)\{\mathcal{R}(0)\}^{n}\right. \\
& \left.\quad+10 n(14-5 n)\{\mathbb{R}(0)\}^{n-1} * \mathbb{R}(\beta)+25 n(n-1)\{\mathbb{R}(0)\}^{n-2} * \mathbb{R}(\beta, \beta)\right\} .
\end{aligned}
$$

This rule is given by Miller [6]. The number of function evaluations it requires is given by (6.4) above and is

$$
\nu\left(E_{2}{ }^{n}(0)\left(G_{3}\right)^{2}\right)=1+2\left(\begin{array}{l}
n \\
1
\end{array}\right)+4\left(\begin{array}{l}
n \\
2
\end{array}\right)=2 n^{2}+1 .
$$

For large $n$, this is considerably less than

$$
\nu\left(\left(G_{3}\right)^{n}\right)=3^{n} .
$$

7. The Rules $\bar{G}_{t+1}^{(n)}$. Instead of making direct use of Theorem 4.2 as it stands, we may proceed in a slightly more involved way to extend the Gauss-Legendre quadrature formula. We consider the integration rule,

$$
R^{(t)}=E_{t-1}^{t}(0)\left(G_{t+1}\right)^{t-1}+\phi\left\{\Re\left(\beta_{1}\right)\right\}^{t}-\phi E_{t-1}^{t}(0)\left(R\left(\beta_{1}\right)\right)^{t-1},
$$

where $\phi$ is an adjustable parameter. We find, without difficulty, that

$$
\odot\left(R^{(t)}\right)=\left(G_{t+1}\right)^{t-1} \text {. }
$$

Now $G_{t+1}$ is of degree $2 t+1$ and so $\left(G_{t+1}\right)^{t-1}$ is also of degree $2 t+1$. Thus 


$$
\left(G_{t+1}\right)^{t-1} f=I^{(t-1)} f \text {, when } f \in \Phi_{t+1}^{(t-1)} .
$$

Using (2.14) and (3.2), together with (7.2), it follows that

$$
R^{(t)} f=I^{(t)} f, \text { when } f \in \Phi_{t+1}^{(t-1)} .
$$

We now choose $\phi$ so that $R^{(t)} f=I^{(t)} f$ when $f$ is the function $x_{1}{ }^{2} x_{2}{ }^{2} \cdots x_{t}{ }^{2}$, that is, the only element of $\phi_{t+1}^{i}$. This is done by simple substitution. We find, without difficulty, that

$$
E_{t-1}^{t}(0)\left(G_{t+1}\right)^{t-1} x_{1}{ }^{2} x_{2}^{2} \cdots x_{t}^{2}=0,
$$

since this rule evaluates this function at points at which it is zero. Moreover,

$$
E_{t-1}^{t}(0)\left\{R\left(\beta_{1}\right)\right\}^{t-1} x_{1}{ }^{2} x_{2}{ }^{2} \cdots x_{t}{ }^{2}=0
$$

for the same reason. $\left\{R\left(\beta_{1}\right)\right\}^{t}$ evaluates this function only at points where all the coordinates are $\beta_{1}$. Thus

$$
\left\{R\left(\beta_{1}\right)\right\}^{t} x_{1}{ }^{2} x_{2}{ }^{2} \cdots x_{\imath}{ }^{2}=\beta_{1}{ }^{2 t},
$$

and the definition $(4.1)$ gives

$$
I^{(t)} x_{1}{ }^{2} x_{2}{ }^{2} \cdots x_{t}{ }^{2}=1 / 3^{t} .
$$

Thus, with this function $f$, the equation $R^{(t)} f=I^{(t)} f$ gives

$$
\phi=1 /\left(3 \beta_{1}{ }^{2}\right)^{t} \text {. }
$$

We define the rule $\bar{G}_{t+1}^{(t)}$ to be $R^{(t)}$ given by (7.1) with $\phi$ taking this value. Thus

$$
\bar{G}_{t+1}^{(t)}=E_{t-1}^{t}(0)\left(G_{t+1}\right)^{t-1}+\frac{1}{\left(3 \beta_{1}^{2}\right)^{t}}\left[\left\{R\left(\beta_{1}\right)\right\}^{t}-E_{t-1}^{t}(0)\left(R\left(\beta_{1}\right)\right)^{t-1}\right]
$$

We have chosen $\phi$ so that

$$
\bar{G}_{t+1}^{(t)} f=I^{(t)} f, \text { when } f \in \phi_{t+1}^{t} .
$$

However, since $\bar{G}_{t+1}^{(t)}$ is a special case of $R^{(t)},(7.4)$ gives

$$
\bar{G}_{t+1}^{(t)} f=I^{(t)} f \text {, when } f \in \Phi_{i+1}^{(t-1)} .
$$

Using (4.7), we see that

$$
\phi_{t+1}^{t} \cup \Phi_{t+1}^{(t-1)}=\Phi_{t+1}^{(t)} .
$$

Thus (7.11) and (7.12) taken together yield

$$
\bar{G}_{t+1}^{(t)} f=I^{(t)} f, \text { when } f \in \Phi_{t+1}^{(t)} .
$$

Thus $\bar{G}_{t+1}^{(t)}$ is of degree $2 t+1$.

We define an $n$-dimensional extension of this rule,

$$
\bar{G}_{t+1}^{(n)}=E_{t}{ }^{n}(0) \bar{G}_{t+1}^{(t)}, \quad n=1,2,3, \cdots .
$$

It is clear that $\bar{G}_{t+1}^{(n)}$ is also of degree $2 t+1$. This rule may be written

$$
\bar{G}_{t+1}^{(n)}=E_{t-1}^{n}(0)\left(G_{t+1}\right)^{t-1}+\frac{1}{\left(3 \beta_{1}\right)^{t}}\left[E_{t}^{n}(0)\left\{\mathcal{Q}\left(\beta_{1}\right)\right\}^{t}-E_{t-1}^{n}(0)\left\{\mathcal{Q}\left(\beta_{1}\right)\right\}^{t-1}\right]
$$


The number of function evaluations required by $\bar{G}_{t+1}^{(n)}$ is

$$
\begin{aligned}
& \nu\left(\bar{G}_{t+1}^{(n)}\right)= 1+t\left(\begin{array}{l}
n \\
1
\end{array}\right)+\cdots+t^{t-1}\left(\begin{array}{c}
n \\
t-1
\end{array}\right)+2^{t}\left(\begin{array}{l}
n \\
t
\end{array}\right) \quad(t \text { even }) \\
&=1+(t+1)\left(\begin{array}{l}
n \\
1
\end{array}\right)+\cdots+(t+1)^{t-1}\left(\begin{array}{c}
n \\
t-1
\end{array}\right) \\
& \quad+2^{t}\left(\begin{array}{l}
n \\
t
\end{array}\right) \quad(t \text { odd }) .
\end{aligned}
$$

This is clearly fewer than the number used by the rule $E_{t}{ }^{n}(0)\left(G_{t+1}\right)^{t}$ of the previous section. That number is given in equation (6.4). In fact, $\bar{G}_{t+1}^{(n)}$ uses as points for function evaluations a subset of the points used by $E_{t}{ }^{n}(0)\left(G_{t+1}\right)^{t}$.

The simplest nontrivial case is that with $t=2$. In this case, $\bar{G}_{3}{ }^{(n)}$ is identical with $E_{2}{ }^{n}(0)\left(G_{3}\right)^{2}$ and is given in $(6.10)$. As an example, we construct $\bar{G}_{4}{ }^{(n)}$. Here

$$
G_{4}=\zeta_{1} R\left(\beta_{1}\right)+\zeta_{2} R\left(\beta_{2}\right)
$$

where

$$
\begin{aligned}
\zeta_{1} & =(18-\sqrt{ } 30) / 36 \\
\zeta_{2} & =(18+\sqrt{ } 30) / 36 \\
{\beta_{1}}^{2} & =(15+2 \sqrt{ } 30) / 35 \\
{\beta_{2}}^{2} & =(15-2 \sqrt{ } 30) / 35 .
\end{aligned}
$$

The term $E_{2}{ }^{n}(0)\left(G_{4}\right)^{2}$ is calculated in the same way as the expression $E_{2}{ }^{n}(0)\left(G_{3}\right)^{2}$ was calculated in Section 6 . We use $(3.13)$ yet again to calculate $E_{3}{ }^{n}(0)\left(\mathcal{R}\left(\beta_{1}\right)\right)^{3}$ and ${E_{2}}^{n}(0)\left(\Re\left(\beta_{1}\right)\right)^{2}$. The final result for (7.16) with $t=3$ is

$$
\begin{aligned}
\bar{G}_{4}^{(n)}= & \{\Re(0)\}^{n}(n-1)(n-2)\left(\frac{1}{2}-\frac{n \phi}{6}\right) \\
& +\{\Re(0)\}^{n-1} * \Re\left(\beta_{1}\right) n(n-2)\left(-\zeta_{1}+\frac{(n-1) \phi}{2}\right) \\
& +\{\Re(0)\}^{n-1} * \Re\left(\beta_{2}\right) n(n-2)\left(-\zeta_{2}\right) \\
& +\{\Re(0)\}^{n-2} * \Re\left(\beta_{1}, \beta_{1}\right) n(n-1)\left(\frac{\zeta_{1}^{2}}{2}-\frac{(n-2) \phi}{2}\right) \\
& +\{\Re(0)\}^{n-2} * \Re\left(\beta_{1}, \beta_{2}\right) n(n-1) \zeta_{1} \zeta_{2} \\
& +\{\Re(0)\}^{n-2} * \Re\left(\beta_{2}, \beta_{2}\right) n(n-1) \frac{\zeta_{2}{ }^{2}}{2} \\
& +\{\Re(0)\}^{n-3} * \Re\left(\beta_{1}, \beta_{1}, \beta_{1}\right) n(n-1)(n-2) \phi / 6,
\end{aligned}
$$

where $\zeta_{1}, \zeta_{2}, \beta_{1}$ and $\beta_{2}$ take the values given in (7.19), and

$$
\phi=1 / 27 \beta_{1}{ }^{6} \text {. }
$$

The rule $\bar{G}_{4}{ }^{(n)}$ is the weighted sum of seven $n$-dimensional basic rules. The number of points required is, therefore, the sum of the number required by each separate 
basic rule and is given by (7.17). Thus

$$
\nu\left(\bar{G}_{4}^{(n)}\right)=1+2.2\left(\begin{array}{l}
n \\
1
\end{array}\right)+4.4\left(\begin{array}{l}
n \\
2
\end{array}\right)+8\left(\begin{array}{l}
n \\
3
\end{array}\right) .
$$

We note that this is an improvement on $E_{3}{ }^{n}(0)\left(G_{4}\right)^{3}$ which uses, in addition, the points required by the basic rules $\{R(0)\}^{n-3} * \mathcal{R}\left(\beta_{1}, \beta_{1}, \beta_{2}\right),\{R(0)\}^{n-3}$ $* \mathscr{R}\left(\beta_{1}, \beta_{2}, \beta_{2}\right)$ and $\{R(0)\}^{n-3} * \mathscr{R}\left(\beta_{2}, \beta_{2}, \beta_{2}\right)$. The expression for $\nu\left(E_{3}{ }^{n}(0)\left(G_{4}\right)^{3}\right)$ differs from (7.22) above, the coefficient of the leading term being larger by a factor of 8 . The rule $\bar{G}_{4}^{(n)}$ is not the most economical. There exists a rule which uses all but one of the seven basic rules used by $\bar{G}_{4}{ }^{(n)}$, the omitted rule being $\mathfrak{R}(0)^{n-2} * \mathbb{R}\left(\beta_{1}, \beta_{2}\right)$. And for certain values of $n$, we have constructed rules of degree 7 which use fewer points than this. These rules will be published in due course.

8. $\nu\left(R_{t+1}^{(n)}\right)$ as $n \rightarrow \infty$. The rules constructed in Section 4 establish a conjecture (Thacher [9]). We state it in the following form:

For fixed $d=2 t+1$, there exists a set of integration rules, $\bar{G}_{t+1}^{(t)}, \bar{G}_{t+1}^{(t+1)}, \cdots$, in $t, t+1, \cdots$ dimensions, each of degree $d$, with the property that

$$
\operatorname{Lim}_{n \rightarrow \infty} \nu\left(\bar{G}_{t+1}^{(n)}\right) / 2^{t}\left(\begin{array}{l}
n \\
t
\end{array}\right)=1 .
$$

Stated in the form of the original conjecture, for symmetric rules of fixed degree $d=2 t+1$ the number of points required goes up as $\left(2^{t} / t !\right) n^{t}$.

The principal purpose of Section 7 is to derive the result stated above. However, the integration rules that we have constructed are themselves of possible practical interest for integration over a high-dimensional hypercube. We exemplify this in the table by listing the number of points required for $n=15$ by the two rules derived here and for the product Gaussian. A lower bound to be derived later is also listed. We shall show in a forthcoming paper that there exist more economical rules for $n=15$ and $d \geqq 3$. These are constructed by a simple extension of the methods in this paper. The important point is that the number of points required, for example, for $d=9$ and $n=15$, is of the order of 50,000 and need not be thirty thousand million. Thus the possibility of carrying out moderate-degree high-dimensional integration using integration rules in practice exists.

These integration rules should be used with caution. The referee has pointed out, quite validly, that the rule $G_{2}{ }^{15}$ requires roughly the same number of points as the rule $\bar{G}_{5}{ }_{5}^{(15)}$ and integrates exactly a majority of the multinomials integrated exactly by $\bar{G}_{5}^{(15)}{ }^{*}$

It may, therefore, easily be the case that use of the more complicated $\bar{G}_{5}{ }^{(15)}$ instead of $G_{2}{ }^{15}$ is not repaid by any appreciable increase in accuracy. The author agrees entirely with this point of view. The intricate question of whether a more accurate result is obtained, in general, by using a high-degree rule or in some other

* The rule $\bar{G}_{b}^{(15)}$ given here uses 52,701 points. However, a similar rule is available which uses only 27,341 points and will be published. This is, in fact, fewer than $\nu\left(G_{2^{15}}\right)$ but the arrangement of the points is much more complicated. Stenger [7] has also derived a rule using 49,061 points. 
way is of the utmost importance. The person carrying out the integration usually wants to know what reliance he may place in his numerical result. This may or may not be related to the degree of the polynomial that the rule will integrate exactly. There is both need for and scope for such an investigation.

The rules derived in this paper may be of use in such a general analysis of integration formulae. However, the purpose of this paper is merely to indicate how rules of particular degree may be constructed.

University of New South Wales

Kensington, N. S. W., Australia

1. C. F. Gauss, "Methodus nova integralium valores per approximationem inveniendi," Comm. Soc. Reg. Sci. Göttingen, 1816.

2. P. C. Hammer, O. J. Marlowe \& A. H. Stroud, "Numerical integration over simplexes and cones," $M T A C$, v. 10, 1956, pp. 130-137. MR 19, 177.

3. P. C. Hammer \& A. H. STroud, "Numerical evaluation of multiple integrals. II," $M T A C$, v. 12,1958, pp. $272-280$. MR $21 * 970$.

4. Z. Kopal, "Numerical analysis," Wiley, New York and Chapman and Hall, London, 1955. MR 17, 1007.

5. J. N. LyNESS, "Symmetric integration rules for hypercubes. I. Error coefficients," Math. Comp., v. 19, 1965, pp. 260-276.

6. J. C. P. Miller, "Numerical quadrature over a rectangular domain in two or more dimensions. Quadrature in several dimensions using special points," Math. Comp., v. 14, 1960, pp. 130-138. MR $22 * 6075$.

7. F. Stenger, "Numerical Integration in n Dimensions," Thesis, University of Alberta, Canada, 1963.

8. A. H. STroud, "Numerical integration formulas of degree 3 for product regions and cones," Math. Comp., v. 15, 1961, pp. 143-150. MR 22 *12717.

9. H. C. Thacher, JR., "Optimum quadrature formulas in $s$ dimensions," $M T A C, \mathrm{v} .11$, 1957, pp. 189-194. 